\title{
The interrelationships of magnetic and magneto acoustic-emission characteristics of heat- treated steels of various chemical composition
}

\author{
Vladimir Kostin ${ }^{1,2 *}$, Evgeniy Serbin ${ }^{1,2}$, and Olga Vasilenko $^{1,2}$ \\ ${ }^{1}$ M.N. Mikheev Institute of Metal Physics of Ural Branch of Russian Academy of Sciences, 620990 \\ Ekaterinburg, Russia \\ ${ }^{2}$ Ural Federal University n.a. B.N. Yeltsin, 620002 Ekaterinburg, Russia
}

\begin{abstract}
The relationship between the amplitude of magnetoacoustic emission and the residual magnetic induction of the materials is shown. The dependence of magnetoacoustic emission (MAE) on the frequency of magnetization reversal of a group of steels of different chemical composition was investigated. In all the investigated cases, the MAE amplitude maximum corresponds to a field frequency of 3-5 Hz. The magnetic properties of two groups of steels subjected to various thermal treatments have been studied. It is shown that the residual magnetic induction of steels is a sufficiently universal parameter for controlling softening thermal treatments and that the amplitude of magnetoacoustic emission can be used as a testing parameter in scanning systems of structurescopy of ferromagnetic steels.
\end{abstract}

\section{Introduction}

At the core of magnetic structurecopy of steel products lies the presence of a correlation between magnetic and hardness properties. However, the use of magnetic testing parameters alone is often not sufficient for complete determination of the structural-phase state of steel products. Additional information can be acquired by parameters related to interaction of moving domain walls with defects of the crystal structure. One of such effects is magnetoacoustic emission (MAE), which is understood as a totality of elastic vibrations that arise in a ferromagnet upon its magnetization reversal.

In the case of low-frequency magnetization reversal of ferromagnets, the main mechanism of MAE is magnetostriction, which manifests itself in a change of the sample size (magnetostriction), as well as in the appearance of local elastic perturbations, mainly caused by irreversible displacements of 90-degree domain walls (magnetic noise). Thus, magnetoacoustic emission makes it possible to register jumps of domain boundaries not only near the surface, but also in the volume of the material $[1,2]$.

The rearrangement of the domain structure upon magnetization reversal can be characterized by various dimensional (area or mean path of the domain boundary), quantitative (the number of simultaneously shifting boundaries), and temporal (time of Barkhausen jump) parameters. The

*Corresponding author: kostin@imp.uran.ru 
MAE signal also depends on the reflection of the elastic vibrations from the boundaries of the ferromagnet and, consequently, the MAE parameters depend on the shape and size of the magnetized object. These features should be reflected in the MAE spectrum, which makes it advisable to use broadband piezoelectric transducers for detection of the MAE instead of the previously used narrow-band $[2,3]$.

The dependence of the MAE on the frequency of the magnetization reversal field is no less important in the analysis of the MAE. The use of the MAE phenomenon in structurescopy implies carrying out a measurement cycle with averaging the amplitude readings over several periods. In addition, the noise nature of the MAE signal and the high sensitivity to noise of an acoustic nature and vibrations requires rapid measurement. This means that the optimal magnetization reversal frequency of a ferromagnet should not be too low, since for a long duration of measuring the field distribution of the MAE amplitude, the amount of interference during the period will increase dramatically [2].

The aim of this work is to establish the relationships between magnetic and magnetoacoustic emission parameters, to establish the correlation of the residual magnetic induction and amplitude of the MAE, to find new testing parameters of ferromagnetic objects in structurescopy, and to determine the dependence of the amplitude of the MAE on the frequency of the remagnetizing field.

\section{Materials and experimental methods}

To study the dependence of magnetoacoustic emission on the frequency of magnetization reversal, samples were made of three grades of steel in the form of plates $4.6 \times 40.1 \times 90$ $\mathrm{mm}$ in size, differing in structural-phase composition and, accordingly, in magnetic properties. The plates were quenched and tempered at various temperatures (Tque and Ttem, respectively). (see Table 1).

Table 1. Characteristics of heat-treated steel plates

\begin{tabular}{|c|c|c|c|c|c|}
\hline Material & $\begin{array}{c}\text { Tque, } \\
{ }^{\circ} \mathbf{C}\end{array}$ & $\begin{array}{c}\text { Ttem, } \\
{ }^{\circ} \mathbf{C}\end{array}$ & $\begin{array}{c}\boldsymbol{B r}, \\
\mathbf{T}\end{array}$ & $\boldsymbol{H c}, \mathbf{A} / \mathbf{c m}$ & $\mathbf{H B}$ \\
\hline Steel 10 & 930 & 400 & 1,1 & 8,9 & 269 \\
Steel 10 & 930 & 500 & 1,3 & 7,9 & 248 \\
Steel 10 & 930 & 600 & 1,5 & 6,0 & 192 \\
65G & 870 & 200 & 0,9 & 32,3 & 601 \\
65G & 870 & 400 & 1,45 & 14,4 & 505 \\
65G & 870 & 600 & 1,55 & 13,6 & 331 \\
95Cr18 & 1070 & 200 & 0,5 & 74,5 & 601 \\
95Cr18 & 1070 & 550 & 0,8 & 28,0 & 534 \\
\hline
\end{tabular}

The magnetic properties of the samples were measured using the REMAGRAPH C-500 measuring system manufactured by Magnet-Physik Steingroever GmbH, Germany. The error in magnetization measuring did not exceed $2 \%$, and the error in field measuring was $1 \%$. The hardness of the samples was measured with a hardness tester TK14-250 with an error of no more than $0.5 \mathrm{HB}$ (Brinell scale).

The effect of the shape and geometric dimensions of ferromagnets on the magnitude and nature of magnetoacoustic emission was studied on samples from annealed permendur $(50 \% \mathrm{Fe}, 50 \% \mathrm{Co})$, from which three samples were made: a short rectangular plate $4.1 \times 40$ $\times 89 \mathrm{~mm}$ (sample P1); a long plate with dimensions of $2 \times 8.9 \times 295 \mathrm{~mm}$ (sample P2) and a shortened plate with dimensions of $2 \times 9 \times 161 \mathrm{~mm}$ (Sample P3).

To evaluate the influence of the stress-strain state on the MAE signal, samples of $20 \mathrm{G}$ and 70G steels were chosen, the stress-strain state of which was varied over a wide range 
by means of cold plastic deformation by rolling up to respectively $40 \%$ and $63 \%$ (by changing the cross-section) and then annealing at various temperatures in the range from 20 to $800{ }^{\circ} \mathrm{C}$ for 1 hour, followed by cooling in air. After the samples were then grinded to remove the dross and the decarburized layer. The final dimensions of the $20 \mathrm{G}$ steel samples were $4 \times 10.2 \times 69 \mathrm{~mm}$, and the $70 \mathrm{G}$ steel was $6 \times 9.5 \times 88 \mathrm{~mm}$. The coercive force of all the samples studied after the grinding has changed insignificantly, which indicates the absence of appreciable plastic deformation during grinding [4].

For excitation of MAE two solenoids with different degrees of field homogeneity were used. The solenoid 1 had a length of $154 \mathrm{~mm}$, a rectangular hole of $30 \times 60 \mathrm{~mm}$, and the field in it was inhomogeneous: at a distance of a quarter of the length from the center, the maximum field amplitude decreased by more than $15 \%$ of the value at the center of the solenoid. The solenoid 2 had a length of $750 \mathrm{~mm}$, a round hole $48 \mathrm{~mm}$ in diameter, and a region of uniform (with an accuracy of $2 \%$ ) field in it was $120 \mathrm{~mm}$. The maximum amplitude of the field in solenoids 1 and 2 reached 160 and $100 \mathrm{~A} / \mathrm{cm}$, respectively. To eliminate extraneous vibrations, the samples in the solenoids were placed either on the foam rubber (solenoid 1) or on the suspension (solenoid 2).

Due to wideness of the spectrum of magnetoacoustic emission (from a few hertz to several megahertz) tested methods and means of acoustic emission testing were used for the measurements. Two broadband acoustic emission sensors LD-11 and A-9 ELTEST (3) with working area diameters of $17.5 \mathrm{~mm}$ (transducer 1) and $21 \mathrm{~mm}$ (transducer 2) were used to record acoustic vibrations. The stability of the acoustic contact was ensured by using Lythol-24 couplant and by fixation of the piezoelectric sensor with an elastic band. In more detail, the setup used for excitation and detection of the MAE is described in $[2,3]$.

\section{Results and discussion}

To determine the character of the dependence of the amplitude of the MAE on the frequency of the magnetization reversal $U_{M A E}\left(f_{r m}\right)$ for a group of samples with different structural-phase states (see Table 1), magnetoacoustic measurements were made on the described setup with solenoid 1 (Fig. 1).

As can be seen from Fig. 1, the presence of a maximum on the dependence of the amplitude of the MAE on the frequency of the alternating field is characteristic for all the samples studied. When the field frequency is reduced below $0.5 \mathrm{~Hz}$, the signal decreases sharply to noise level. As the frequency increases, the signal rapidly decreases and at a frequency of $15 \mathrm{~Hz}$ or more it also becomes commensurate with the noise level. The nonmonotonicity of the dependence of the amplitude of the MAE on the frequency of the alternating field and the "resonant" frequency of the MAE occurring at frequencies of 3-4 Hz are observed for all samples of the group, despite the differences in their physical properties.

The measurement of magnetoacoustic emission in an inhomogeneous field has practical importance, since in the future a local testing of the material properties is assumed. However, in a nonuniform magnetic field, the ponderomotive force is additionally affects ferromagnetic samples. 


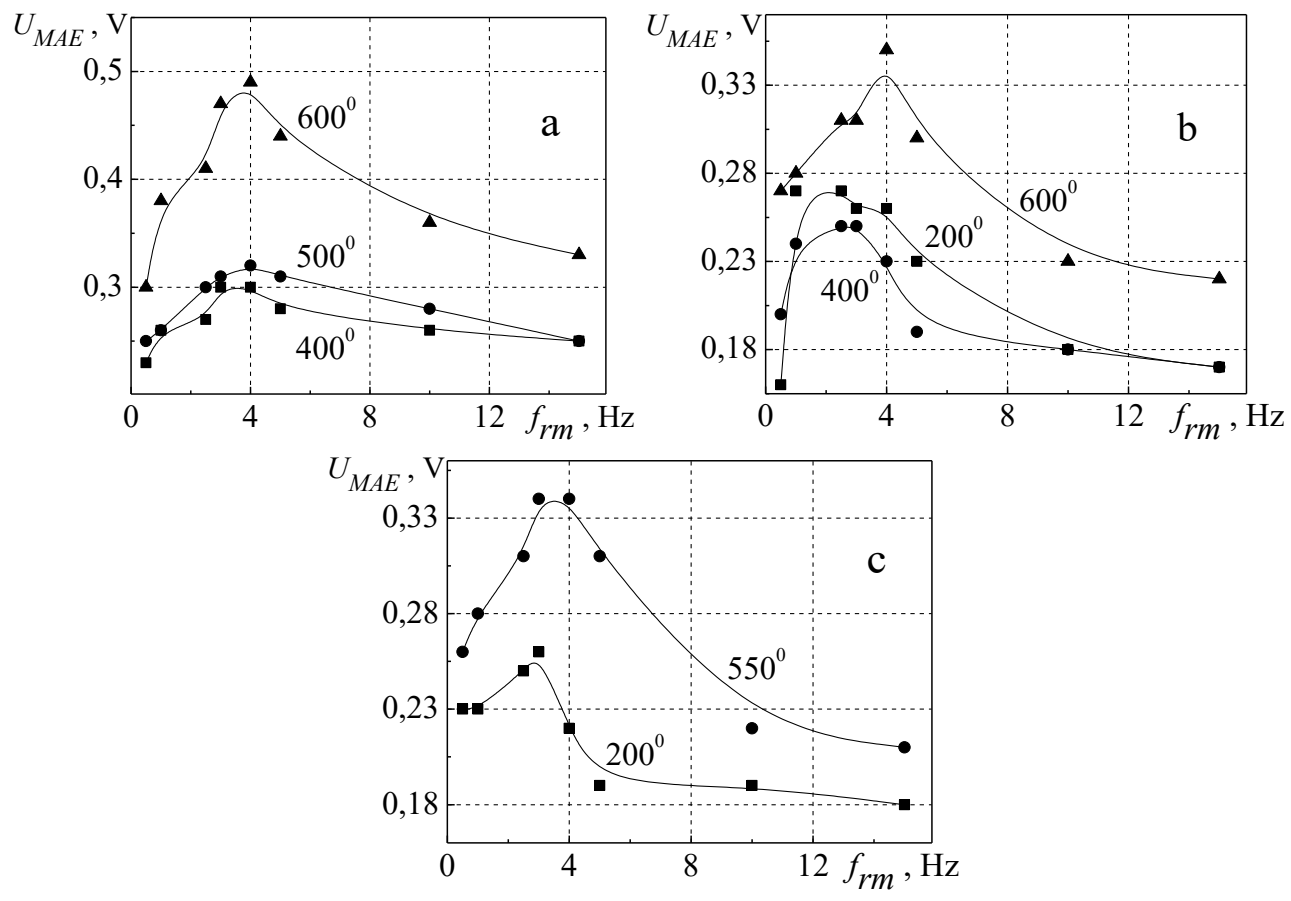

Fig. 1. Dependences of MAE amplitude of quenched and tempered at different temperatures samples of (a) steel 10, (b) steel $65 \mathrm{G}$ and (c) steel $95 \mathrm{Cr} 18$ on frequency of the field $f_{r m}$. Tempering temperatures of particular sample shown on the figure.

It is known that the coercive force $H c$ decreases monotonically with increasing tempering temperature of more than $300{ }^{\circ} \mathrm{C}$ only for hardened steels with a carbon content of less than $0.3 \%$ (see the properties of steel 10 in Table 1). With a higher carbon content, the coercive force at medium and high temperature tempering varies little or even ambiguously. However, with an increase in tempering temperature from $300{ }^{\circ} \mathrm{C}$ and higher for steels with different carbon content, monotonic growth of the residual magnetic induction of substance $\mathrm{Br}$ is noticeable (by a factor of 1.5 - 2), which is also evident from Table. 1.

A decrease in the coercive force $\mathrm{Hc}$ and an increase in the residual magnetic induction $\mathrm{Br}$ also take place during softening of the cold-deformed steels as a result of their annealing. Dependences of the residual magnetic induction and coercive force on the annealing temperature of $20 \mathrm{G}$ and $70 \mathrm{G}$ steels are shown in Fig. 2.

The coercive force of these steels decreasing, starting at Tann $=100{ }^{\circ} \mathrm{C}$. The drop in $H c$ in steel $20 \mathrm{G}$ occurs over the entire annealing temperature range of the samples.

The $H c$ (Tann) dependence for steel $70 \mathrm{G}$ has a local maximum at Tann $\sim 600{ }^{\circ} \mathrm{C}$, the presence of which is explained by the coagulation of carbides, when the size of the inclusions becomes commensurate with the thickness of the interdomain boundary. A weak and ambiguous change in the coercive force at high annealing temperatures does not allow the use of its value as a quality testing parameter for the annealing of the investigated steels.

The residual magnetic induction $\mathrm{Br}$ is monotonically increasing to $\sim 700{ }^{\circ} \mathrm{C}$. The change in the magnetic parameters at temperatures below $(450-500){ }^{\circ} \mathrm{C}$ is associated with the removal of distortions of the crystal lattice and the yield of carbon from the lattice of the supersaturated $\alpha$ solid solution, and at higher temperatures - recrystallization processes occur. The decrease in $\mathrm{Br}$ at Tann above $700{ }^{\circ} \mathrm{C}$ is due to the deterioration of the structure and the increase in the level of internal stresses due to rapid cooling on air [3]. 

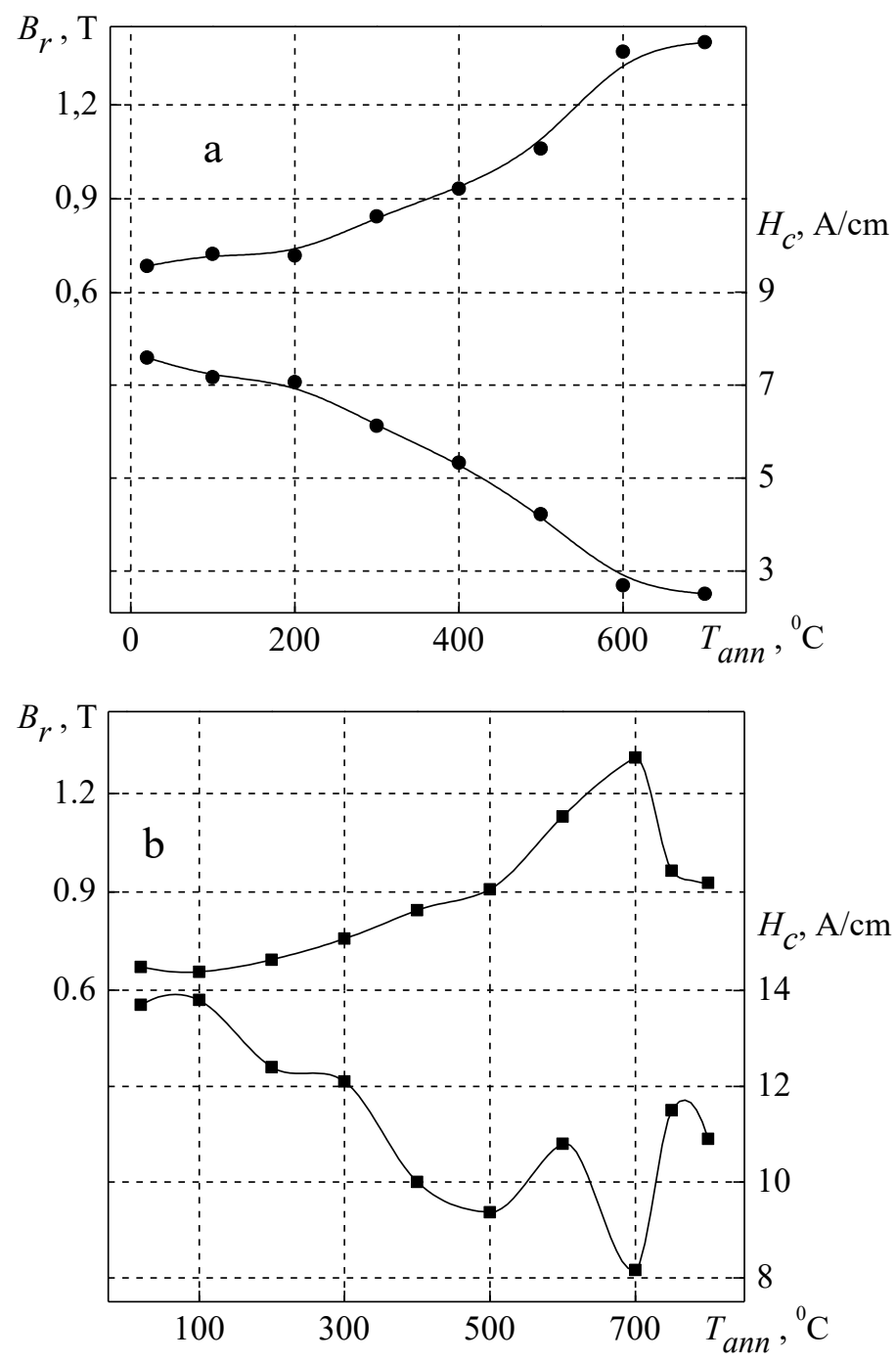

Fig. 2. Dependences of residual magnetic induction and coercive force on annealing temperature of steels 20G (a) and 70G (b)

Dependences of the residual magnetic induction $\mathrm{Br}$ on the hardness of cold-deformed and annealed at different temperatures samples from steels $20 \mathrm{G}$ and $70 \mathrm{G}$ are shown in Fig. 3.

With an increase of the annealing temperature of $20 \mathrm{G}$ steel in the temperature range from 300 to $700{ }^{\circ} \mathrm{C}$, an increasing drop in hardness occurs, while the residual magnetic induction on the whole range not changing so intensively, especially at high annealing temperatures, where it is practically constant (Fig. 3a). This leads to a nonlinear dependence of the residual magnetic induction on the hardness and, accordingly, to a loss of sensitivity of $\mathrm{Br}$ to hardness as a testing parameter. Thus, for steel $20 \mathrm{G}$, it is necessary to search for new hardness testing parameters. For steel 70G, the residual magnetic induction decreases linearly with increasing hardness and the value can be a hardness testing parameter. 

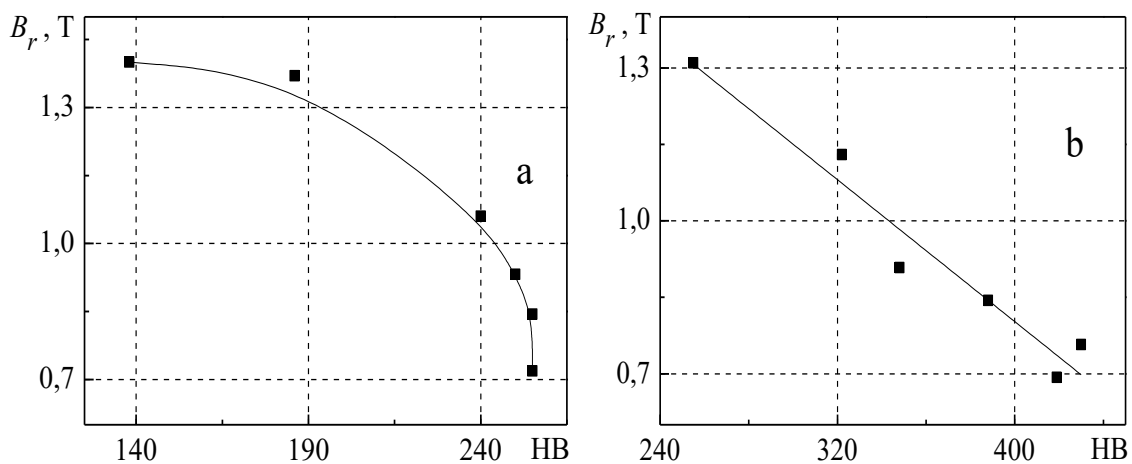

Fig. 3. Dependences of residual magnetic induction on hardness of samples of cold-deformed and annealed steels $20 \mathrm{G}$ (a) and $70 \mathrm{G}$ (b)

From the foregoing it follows that it is relevant to search for rapidly measured parameters of structurescopy suitable for use in scanning diagnostic systems. To this end, the parameters of magnetoacoustic emission were investigated on samples of these steels $20 \mathrm{G}$ and $70 \mathrm{G}$.

On Fig. 4 shown the dependences of the amplitude of the MAE $\left(U_{M A E}\right)$ and the residual magnetic induction on the annealing temperature of samples from cold-deformed and annealed $20 \mathrm{G}$ and $70 \mathrm{G}$ steels. In Fig. 4 is clearly seen correlation between the residual magnetic induction and the amplitude of the MAE.
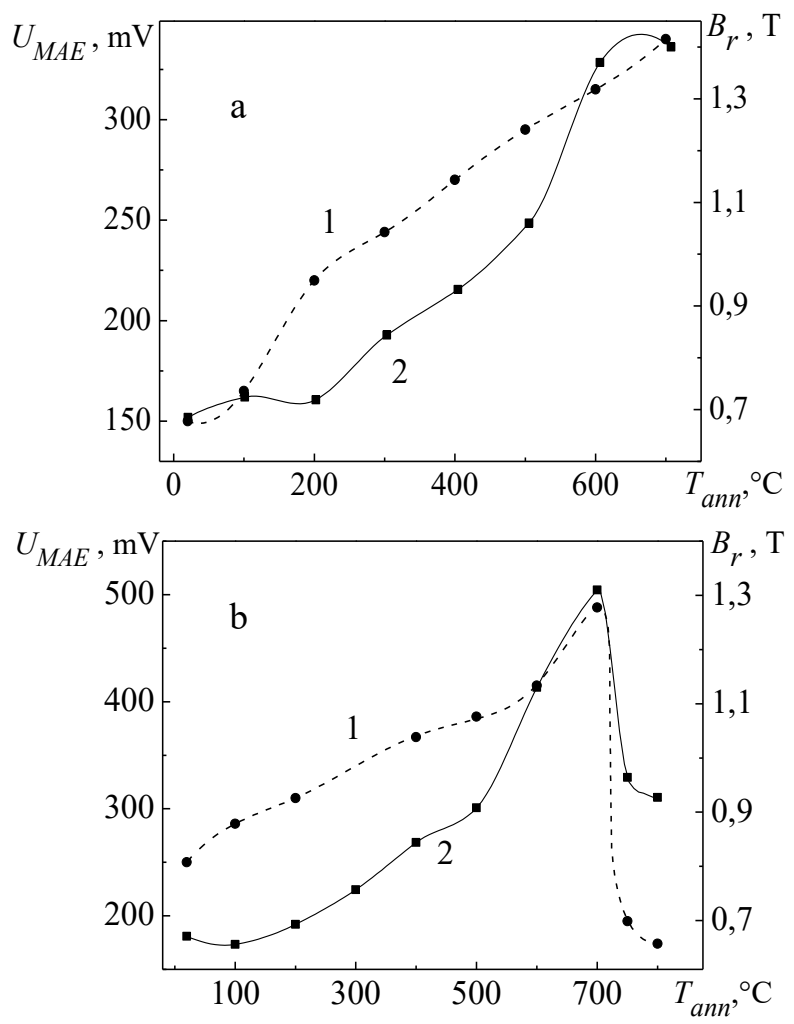

Fig. 4. Dependences of MAE amplitude (1) and residual magnetic induction (2) on temperature of annealing of samples of cold-deformed and annealed steels $20 \mathrm{G}$ (a) and $70 \mathrm{G}$ (b) 
In particular, the dependence of the amplitude of magnetoacoustic emission on the annealing temperature of steel $70 \mathrm{G}$ accurately reproduces the maximum of the dependence of the residual magnetic induction on the annealing temperature. With the subsequent increase in Tann, the MAE signal is rapidly damped due to the deterioration of the steel structure.

\section{Conclusion}

It is established that the dependence of the amplitude of magnetoacoustic emission on the frequency of the magnetizing field has nonmonotonic character. For a large group of different in physical properties and chemical composition steels, the field frequency corresponding to the maximum MAE amplitude is $3-5 \mathrm{~Hz}$.

The existence of a relationship between the residual magnetic induction of matter and the amplitude of magnetoacoustic emission of annealed steels $20 \mathrm{G}$ and $70 \mathrm{G}$ is established, which consists in correlation of the dependences of these parameters on the annealing temperature of steels. The value of $U_{M A E}$ along with the residual magnetic induction can be a parameter in the scanning systems of structurescopy of ferromagnetic steels.

The work is carried out under the program of fundamental research of UrB of RAS 2015-2017, Project 15-17-2-5.

\section{References}

1. V.N. Kostin., V.I. Pudov, E.D. Serbin, O.N. Vasilenko, J. Deform. Fract. Mater. 2, 41 (2017) (on Russian)

2. V.N. Kostin, D.Yu. Filatenkov, Yu.A. Chekasina, O.N. Vasilenko, E.D. Serbin, Acoust. Phys. 2, 237 (2017)

3. V.N. Kostin, M.A. Guriev, O.N. Vasilenko, D.Yu. Filatenkov, Y.G. Smorodinsky, Phys. Mesomech. 16, 103 (2013)

4. V.N. Kostin, O.N. Vasilenko, D.Yu. Filatenkov, Yu.A. Chekasina, E.D. Serbin, Russ. J. Nondestr. Test. 51, 624 (2015) 